\section{$\underset{\substack{\text { hommes } \\ \text { \& migrations }}}{ }$}

\section{Hommes \& migrations}

Revue française de référence sur les dynamiques

migratoires

1299 | 2012

Musulmanes et féministes en Grande-Bretagne

\title{
Les musulmans dans la société britannique
}

\section{Danièle Joly}

\section{OpenEdition \\ Journals}

Édition électronique

URL : http://journals.openedition.org/hommesmigrations/1402

DOI : 10.4000/hommesmigrations. 1402

ISSN : 2262-3353

\section{Éditeur}

Musée national de l'histoire de l'immigration

Édition imprimée

Date de publication : 1 septembre 2012

Pagination : 12-22

ISSN : 1142-852X

Référence électronique

Danièle Joly, "Les musulmans dans la société britannique », Hommes \& migrations [En ligne], 1299 | 2012, mis en ligne le 31 décembre 2014, consulté le 30 avril 2019. URL : http://

journals.openedition.org/hommesmigrations/1402; DOI : 10.4000/hommesmigrations.1402 


\section{Les musulmans dans la société britannique ${ }^{(1)}$}

Par Danièle Joly, Institut d'études avancées (Paris), Marie Curie fellow au CADIS-EHESS, professeure émérite, université de Warwick

La Grande-Bretagne comptait plus d'un million et demi de musulmans en 2001, principalement originaires de la péninsule indienne. Dans cette société multiculturelle et multiconfessionnelle, l'islam a pignon sur rue et les associations musulmanes participent au dynamisme de la société civile. Les attentats de 2001 à New York et de 2005 à Londres ont brouillé ces relations. Dorénavant, si les pouvoirs publics souhaitent ne pas couper le contact avec ces communautés, ils entendent les garder sous surveillance. 
Les populations de pays de tradition musulmane en Grande-Bretagne n'ont revendiqué que tardivement leur identité religieuse. Dans les premiers temps de leur installation, elles furent identifiées suivant des catégories d'abord fondées sur les relations raciales, puis selon des qualificatifs ethniques, Asian (Asiatiques) se rapportant aux originaires de la péninsule indienne, enrichis de subdivisions ethno-nationales plus précises, telles que Pakistanais, Bangladeshis, etc. Ces catégories correspondaient à la formulation des politiques publiques ainsi qu'aux modes de mobilisation qui se déclinaient pour la défense des intérêts des populations musulmanes à des périodes données. Quant aux musulmans, ils ont d'abord édifié des réseaux et des institutions qui reflétaient leurs différences ethno-nationales et théologiques sans formuler de projet ni d'identification communs, tandis que les politiques publiques ne les distinguaient pas en tant que tels. À l'heure actuelle, au contraire, musulmans et islam occupent le devant de la scène tant par leurs revendications que par l'attention que leur a accordée l'État en Grande-Bretagne, où il n'est pas exagéré de postuler une institutionnalisation de l'islam. Les raisons qui ont amené une différenciation sur la base de l'islam sont complexes et mettent en jeu des facteurs qui tiennent à la fois à la conjoncture internationale, au contexte national et à la situation particulière des musulmans en Grande-Bretagne.

À la charnière des années 1989-1991, un événement historique a fait basculer l'équilibre des forces internationales : l'effondrement des régimes communistes a inauguré l'hégémonie indiscutée des États-Unis et de son modèle néolibéral dans le monde. La fin du projet socialo-communiste a créé un vide qui a facilité une revitalisation de la religion. Les pays occidentaux substituent de plus en plus l'islam au communisme, en lui décernant le titre d'ennemi public numéro un à la faveur d'événements comme l'accès de talibans au pouvoir en Afghanistan et les attentats à New York en septembre 2001, ainsi qu'en Grande-Bretagne en 2005 : le discours de Tony Blair en août 2005 est révélateur, qui attribue à l'islam radical "les mêmes caractéristiques que le communisme révolutionnaire" (The Guardian, 5 août 2005). L'Occident a été conforté dans cette opinion par des attentats spectaculaires de la main de musulmans. Entre-temps, l'islam avait commencé à se ménager un espace dans la société britannique en mettant à profit l'opportunité que représentait l'existence d'une religion d'État.

Les données du dernier recensement de 2011 n'ayant pas encore été analysées, nous devons nous contenter de celles du recensement de 2001. Celui-ci, comportant une question sur la religion, nous livre le chiffre de 1,6 million de musulmans en GrandeBretagne, c'est-à-dire à peu près $3 \%$ de la population; ce chiffre a progressé de 21000 en 1951 et de 600000 en 1981, jusqu'à 1 million en 1991. Il s'agit d'une population très diversifiée. Un million de personnes sont originaires de la péninsule indienne, 
dont environ les deux tiers sont de souche pakistanaise, un tiers bangladeshie, et le reste d'origine indienne. Elles constituent la dernière vague de l'immigration de travail de l'après-guerre. D'autres viennent de Chypre, du Moyen-Orient, d'Afrique, de l'Europe de l'Est et de l'Extrême-Orient, dont 60000 nées en Europe de l'Est (principalement au Kosovo et en Bosnie), 93000 au Moyen-Orient et 36000 en Afrique du Nord. Parmi ces groupes, de nombreux réfugiés, arrivésplus récemment, relèvent d'un phénomène migratoire différent. La population musulmane compte la moyenne d'âge la plus jeune, puisqu'un tiers a moins de 15 ans, contre une moyenne nationale de $20 \%$, et que $50 \%$ a moins de 24 ans, contre une moyenne nationale de 30 \%. La majorité de cette population est née en Grande-Bretagne. Une forte concentration géographique caractérise les musulmans qui se regroupent à Londres (1 million), dans les West Midlands (192 000), dans la région de Manchester (125 000) et dans les villes du West Yorkshire au nord de l'Angleterre $(150 \text { 000) })^{(2)}$. Les musulmans sont aussi caractérisés par une forte ségrégation dans certains quartiers qui s'explique tout d'abord par la structure de l'emploi et du logement, puis par la migration en chaîne et l'endogamie.

\section{Des populations désavantagées}

Les musulmans (en majorité d'origine pakistanaise et bangladeshie) font partie des populations qui n'ont pas réussi à bénéficier des politiques publiques autant que certaines autres, comme les minorités ethniques indiennes. Cela est corroboré par tous les indicateurs de pauvreté signalant qu'ils restent à l'heure actuelle les plus désavantagés d'après le recensement national de 2001 et sa question sur la religion. Le taux de chômage de la population musulmane est trois fois plus élevé que la moyenne. De plus, elle occupe les emplois les plus mal payés, trois fois plus que la moyenne également. Alors que la moyenne nationale compte $26 \%$ de travailleurs dans les emplois à "col blanc", on n'y trouve que 13 \% de musulmans. Quant aux postes les plus élevés, moins de 3 \% de musulmans les occupent, c'est-à-dire deux fois moins que la population dans son ensemble (6,1 \%). Les écarts sont frappants chez les Pakistanais et les Bangladeshis : les hommes gagnent entre $5000 £$ et $6500 £$ de moins que les Blancs (quasiment tous chrétiens ou agnostiques) à qualifications égales. Un enfant pakistanais et bangladeshi sur trois vit en dessous du seuil de pauvreté, un sur cinq chez les autres. Un candidat musulman a trois fois moins de chances d'obtenir le poste qu'il brigue que les autres ${ }^{(3)}$. Il n'y a pas à s'y tromper: tous les indices de pauvreté relèguent les musulmans dans les derniers échelons socio-économiques. 


\section{Modes d'organisation}

La reconnaissance des communautés musulmanes et de leurs associations est le fruit de l'interaction particulière entre les institutions britanniques et les minorités ethniques. En premier lieu, rappelons-le, la Grande-Bretagne n'est pas constituée d'une république "une et indivisible" comme la France, par exemple ; au contraire, il s'agit d'un Royaume-Uni composé de plusieurs nations, autrement dit, c'est une communauté de communautés, comme l'affirme lord Parekh dans son rapport sur le multiculturalisme ${ }^{(4)}$. D'autre part, l'histoire coloniale de la Grande-Bretagne l'a accoutumée à un modèle d'interaction avec ses sujets colonisés qui mettait à profit l'existence de structures et de leaders indigènes pour mieux gouverner l'Empire. La gestion des populations immigrées a tout naturellement reproduit en partie ce modèle ${ }^{(5)}$.

Par ailleurs, un certain nombre de facteurs a facilité la reconstitution de communautés et d'associations. La structure de l'emploi et du logement a posé les bases matérielles du

\section{Les institutions religieuses sont prépondérantes au sein de la vie associative parmi les populations de pays de tradition musulmane.} regroupement dans les quartiers les plus délabrés à proximité des centres-villes, tendance centripète renforcée par la migration en chaîne. Les musulmans originaires de la péninsule indienne ont, en partie, recréé leurs réseaux familiaux étendus et leurs réseaux villageois parmi lesquels se détachent quelques grands groupes ethniconationaux ${ }^{(6)}$. Dans certaines villes, existe aussi une petite communauté de Yéménites et de Chypriotes turcs, auxquels il faut ajouter des réfugiés et des demandeurs d'asile comme les Bosniaques, les Somaliens, tous ceux qui ont fui le Moyen-Orient ou l'Afrique du Nord. Leurs institutions se sont reconstituées, ainsi que les commerces et les associations dédiées aux populations musulmanes. Dans l'ensemble, la scène est dominée par les musulmans originaires de la péninsule indienne qui sont les plus nombreux et les premiers installés. L'islam britannique est un islam "Asian".

Les institutions religieuses sont prépondérantes au sein de la vie associative parmi les populations de pays de tradition musulmane. Ce sont les mosquées et les associations islamiques qui ont orienté le mode d'incorporation des populations musulmanes dans la société d'accueil. Les mosquées sont nombreuses et variées. Environ 1000 lieux de culte musulman sont enregistrés officiellement en Grande-Bretagne, mais ils sont en réalité beaucoup plus nombreux. L'existence en Grande-Bretagne d'une société civile foisonnante d'associations et d'activités a sans aucun doute favorisé l'émergence de multiples associations parmi les musulmans. L'importance de la société civile dans la 
gestion des affaires publiques confere aux associations une influence significative dans leur interaction avec l'État.

\section{Les modalités de l'action politique}

En Grande-Bretagne, un accident "historique", la loi sur la nationalité et le Commonwealth de 1948, qui n'avait pas été envisagée pour traiter la question des immigrés du nouveau Commonwealth, a pourtant été déterminant à leur endroit. L'ancien Commonwealth est composé des plus anciennes colonies de peuplement britanniques : l'Australie, la Nouvelle-Zélande et le Canada, tandis que le nouveau Commonwealth comprend les colonies ayant obtenu l'indépendance après la Deuxième Guerre mondiale, comme l'Inde, le Pakistan, plusieurs États africains, la Jamaïque, Trinidad, etc. L'appartenance au Commonwealth leur a octroyé tous les droits politiques et sociaux dès leur installation au Royaume-Uni, en tant que citoyens britanniques en vertu de la loi sur la nationalité et le Commonwealth de 1948. Bien que l'exercice et la pleine jouissance de ces droits n'aient été réalisés qu'au prix d'efforts et de pressions multiples, il n'en reste pas moins que ces dispositions ont eu des répercussions décisives. La porte était ouverte à la participation politique des immigrés qui s'en saisirent pour défendre leurs intérêts. À long terme, il en a résulté une interaction intense entre les institutions majoritaires et les immigrés, ainsi qu'une culture de la participation chez ces derniers. La liberté d'association dépourvue d'entraves administratives pour les Britanniques comme pour les étrangers a encore stimulé ce processus. Les immigrés, qui étaient partie prenante et pouvaient influencer les politiques les concernant n'ont pas manqué de le faire en s'investissant dans les partis britanniques, dans le processus électoral et la politique locale par leur action individuelle ou organisée ${ }^{(7)}$.

Toutes les activités politiques légales sont donc accessibles sans restrictions aux musulmans du Commonwealth. Ces dispositions ne s'appliquent pas aux nombreux nouveaux immigrés qui ne sont pas originaires du Commonwealth, mais la majorité des musulmans installés en Grande-Bretagne en étant issus en bénéficient. Quant aux enfants nés de parents étrangers, ils acquièrent aujourd'hui la nationalité britannique automatiquement si l'un des parents est détenteur d'un permis de résidence à long terme. La représentation musulmane dans les institutions de l'État n'est pas négligeable au niveau local, puisqu'elle peut se réclamer de 230 conseillers municipaux en 2010. Les musulmans sont moins visibles au niveau national mais, en 2011, ils comptent 10 membres de la Chambre des lords, 9 députés et 2 députés européens. 


\section{L’islam et l'État}

Pour bien comprendre la nature des rapports entre les populations musulmanes et l'État en Grande-Bretagne, il faut d'abord souligner les particularités de la situation britannique. La Grande-Bretagne est une société chrétienne protestante, non seulement par son histoire et ses traditions, mais aussi par ses structures étatiques. La reine cumule les fonctions officielles de chef de l'Église anglicane, Church of England, et de l'État, même si cela reste symbolique. Deux exemples suffiront à illustrer cette situation. Les évêques siègent ex officio à la Chambre des lords. Les lois sur l'Éducation de 1944 et de 1988 exigent que toutes les écoles enseignent la religion, tandis que la loi de 1988 insiste sur le caractère chrétien de la prière collective quotidienne obligatoire. Les écoles d'État confessionnelles comprennent protestants, catholiques, juifs, sikhs, hindous et musulmans. Le clergé est même investi de certaines prérogatives de l'État, puisque les pasteurs anglicans sont habilités à célébrer des mariages qui sont automatiquement légaux sans passer par le Registry Office (l'équivalent de la mairie en France), dispositions qui s'appliquent aux catholiques et aux juifs. Autrement dit, la religion a droit de cité dans l'espace public britannique ${ }^{(8)}$. Cela a octroyé aux musulmans des justifications morales et légales pour appuyer leurs revendications et rehausser leurs chances de succès. C'est ce qu'ils ont fait avec détermination.

Une autre caractéristique de l'État britannique a un impact déterminant sur les modalités de ses rapports avec les musulmans : une forte décentralisation des pouvoirs subsiste, malgré les efforts du gouvernement conservateur pour la réduire. La plupart des questions soulevées par les musulmans sont traitées par les municipalités au niveau local. En conséquence, la mobilisation locale des musulmans a obtenu des résultats significatifs tenant compte de leurs revendications sur les questions de carrés musulmans dans les cimetières, sur la construction de mosquées, les écoles, les prisons, les hôpitaux, les repas dans toutes les instances publiques, etc ${ }^{(9)}$.

\section{La création d'associations nationales et l'engagement citoyen}

Il est indéniable que les musulmans occupent l'espace public tant par leur militantisme que par la reconnaissance de l'État. La première grande mobilisation nationale a eu lieu à propos de l'Education Reform Act de 1988 et a été suivie en 1989 par les manifestations contre les Versets sataniques de Salman Rushdie, ce qui a motivé la création d'un premier comité national. Avec les encouragements du Parti travailliste dès 1994, cela a débouché en 2004 sur la création du Muslim Council of Britain (MCB, 
Conseil musulman de Grande-Bretagne) regroupant 500 associations affiliées en $2012^{(10)}$. Une collaboration suivie entre le gouvernement et le MCB a pu aussi s'engager. Le MCB a démontré sa volonté de participer aux mécanismes politiques en insistant sur le devoir de voter et en appelant à un vote en faveur du Parti travailliste en 2001 et en 2005, malgré l'intervention de la Grande-Bretagne en Irak ${ }^{(11)}$.

Les jeunes musulmans se sont longtemps montrés discrets sur la scène publique britannique. Alors que les anciens ont intégré la pratique de la religion aux traditions et aux réseaux ethniques liés au lieu d'origine, les jeunes remettent en question ce modèle et se réclament d'un islam plus moderne, universaliste, dépouillé de ses attaches ethniques ${ }^{(12)}$. Ils ont fondé bon nombre d'associations, dont seulement une infime minorité s'est engagée dans un militantisme isolationniste.

Les jeunes musulmanes se distinguent de leurs pairs masculins. Les plus remarquées sont celles que la guerre en Afghanistan et en Irak a projetées sur le devant de la scène de façon inattendue. La campagne contre la guerre (Stop the War Coalition) a été lancée par l'opposition de gauche au gouvernement travailliste, et principalement par des organisations trotskistes. Néanmoins, elle a ouvert aux musulmans dans leur ensemble de nouvelles voies de participation en intégrant à la fois des organisations musulmanes (le MAB et le Muslim Parliament) et des musulmans à titre personnel, des jeunes dont beaucoup de jeunes femmes ${ }^{(13)}$. La présidente de la campagne, notamment, est une jeune femme de culture musulmane, Salma Yaqoob, et plusieurs d'entre elles sont secrétaires de sections locales ou régionales. La campagne a réussi à susciter une mobilisation considérable, y compris une manifestation de 2 millions de personnes contre la guerre en Irak le 15 février 2003 à Londres, la plus grande manifestation que la ville ait jamais connue. Une nouvelle communauté morale s'est constituée audelà des distinctions ethniques et religieuses. Ces musulmans font indubitablement la démonstration de leur participation et de leur citoyenneté.

\section{Les attentats : New York 9/11, Londres 7/7}

Les attentats de New York, le 11 septembre 2001, avaient bouleversé le gouvernement et la population britanniques qui, en général, se sentent proches des États-Unis. Mais la Grande-Bretagne a elle-même été frappée le 7 juillet 2005, lorsque Londres fut la victime d'attentats suicides dans le métro et dans un bus. Le bilan total s'est élevé à plus de 50 morts et 700 blessés. La stupeur et l'incrédulité furent manifestes tant parmi la population majoritaire et le gouvernement que parmi les musulmans.

La première conséquence immédiate des attentats suicides fut l'augmentation des actes de violence verbale et physique contre les Asiatiques (Asians) et surtout contre les musulmans. Cette nouvelle forme de racisme, l'islamophobie décrite par la Commission 
on British Muslims and Islamophobia (1997), n'a pas été enfantée par les attentats suicides mais ceux-ci ont largement contribué à l'alimenter. L'islamisme radical personnifié par Oussama Ben Laden a projeté une image de l'islam particulièrement négative. Les musulmans britanniques en ont fait les frais. L'amalgame entre islam et terrorisme intensifie cette islamophobie. Bien que l'islamophobie puisse se manifester de façon tout aussi virulente et pernicieuse que le racisme classique, il est moins aisé de la contrer car les instruments légaux s'y prêtent moins. En effet, la loi sur les relations raciales de 1976 ne retient pas la religion comme critère de discrimination. Il est vrai que le Human Rights Act de 1998 (la loi sur les droits de l'homme) et une directive de l'Union européenne sur l'emploi interdisent la

\section{Les attentats de New York, le 11 septembre 2001, avaient bouleversé le gouvernement et la population britanniques qui, en général, se sentent proches des États-Unis. Mais la Grande- Bretagne a elle-même été frappée le 7 juillet 2005, lorsque Londres fut la victime d'attentats suicides dans le métro et dans un bus.} discrimination sur des critères religieux.

Cette dernière a été incorporée au corpus législatif britannique par l'Employment Equality Act (2003) pour l'emploi et par l'Equality Act pour tous les services et institutions publics, mais ce sont des instruments légaux plus faibles que la loi de 1976, elle-même renforcée par l'amendement de 2000. Les musulmans eux-mêmes ont fait part de leur anxiété face aux réactions de la société britannique dans le sillage des attentats et aux marques d'hostilité accrues. Les musulmans ont exprimé leur entière désapprobation et leur incompréhension face aux attentats. Leur réaction fut immédiate : une heure ne s'était pas écoulée après les explosions que les associations musulmanes prononçaient une condamnation formelle des attentats. Le 18 juillet 2005, plus de 500 leaders musulmans ont émis une fatwa intitulée "Pas en notre nom", dans laquelle ils condamnaient les attentats de Londres comme allant à l'encontre des enseignements de l'islam, tout en exprimant leur détermination "à cuvrer avec la société majoritaire pour extirper toute forme d'extrémisme en Grande-Bretagne ${ }^{(14) ”}$.

\section{Le gouvernement, les musulmans et la politique antiterrorisme}

En réponse aux attentats de Londres, le 7 juillet 2005, le gouvernement a développé une double stratégie, dans la droite ligne de la politique élaborée après ceux de New York, le 11 septembre 2001 : dans l'immédiat, ne pas stigmatiser les musulmans de Grande-Bretagne tout en appuyant inconditionnellement les initiatives des États-Unis 
dans leur effort de guerre contre la "terreur". Le ministre de l'Intérieur a mis en place sept groupes de travail qui comprenaient une représentation musulmane afin de promouvoir le processus d'intégration et de lutter contre le terrorisme. Leur cahier des charges couvrait les questions suivantes: ouvrir le dialogue avec les jeunes ; lutter contre l'extrémisme et la radicalisation ; appuyer les initiatives communautaires au niveau local et régional ; ouvrir le dialogue avec les femmes ; faciliter la formation et la qualification des imams, soutenir les mosquées en tant que ressources pour les communautés ; développer des initiatives dans l'éducation pour répondre aux besoins des communautés; assurer la sécurité des communautés. Dans son rapport, le Preventing Extremism Together' Working Groups met l'accent sur l'importance de mesures dans le domaine de l'éducation pour "assurer une représentation fidèle de l'islam et de sa civilisation dans le système éducatif' et pour "améliorer la réussite scolaire des élèves musulmans ${ }^{(15)}$ ".

Le deuxième volet de la politique gouvernementale est celui d'un durcissement. En 2005 , le discours de Tony Blair annonce la fermeté : "la règle du jeu a changê(16)" et des mesures de plus en plus draconiennes contre le "terrorisme" sont introduites, renchérissant sur celles qui avaient été engagées après les attentats de New York. La loi sur le terrorisme de 2001 (Anti-Terrorism Crime and Security Act) est renforcée par deux nouvelles lois : le Prevention of Terrorism Act (2005) et le Terrorism Act (2006), qui compte environ 200 mesures juridiques antiterroristes, dont une extension de la période de détention sans inculpation. La détention de 28 jours maximum en garde à vue a été particulièrement critiquée au nom de la Convention européenne des droits de l'homme, de l'Habeas Corpus Act de 1679 et du Bill of Rights de 1689. Cette entorse à l'Habeas Corpus, qui constitue un mythe fondateur et le socle du droit britannique, a soulevé bien des protestations. Les critiques ont détecté un exercice de funambule de la part du gouvernement, qui semblait s'évertuer à mener une guerre contre les musulmans à l'étranger, tout en s'efforçant de ne pas perdre leurs votes et leur appui en Grande-Bretagne. Mais les jeunes hommes musulmans sont de toute évidence devenus la cible d'un harcèlement de la part de la police, constituant la large majorité des personnes arrêtées lors des manifestations pacifiques comme celles contre la English Defence League (EDL), une organisation néo-fasciste. De plus, les arrestations et gardes à vue prolongées sans inculpation de jeunes musulmans se multiplient à la faveur des lois contre le terrorisme.

Dans la pratique, la Grande-Bretagne est une société multiculturelle et le multiculturalisme constitue la doctrine officielle adoptée par le gouvernement pour gérer les minorités ethniques. Malgré les initiatives et discours gouvernementaux qui discréditent le multiculturalisme et promeuvent la "cohésion communautaire" et la "cohésion sociale", les politiques mises en place ont de facto infléchi le multiculturalisme vers un multiconfessionalisme qui s'adresse en particulier aux musulmans. Dans la foulée des attentats, des mécanismes de consultation ont été mis sur pied au niveau 
local mais aussi national avec les jeunes et les femmes de communautés musulmanes. Par exemple, le programme anti-extrémisme Prevent Violent Extremism PathFinder Fund (Prevent) a permis de financer un grand nombre d'associations et de projets musulmans gérés par les municipalités, et dont les modalités d'interaction avec les musulmans se sont calquées sur le modus operandi de la politique multiculturaliste. La majorité des associations en ayant bénéficié sont notamment des associations de femmes et de jeunes de pays de tradition musulmane. Cela a favorisé leur multiplication. Néanmoins, le programme Prevent reste très controversé, étant donné ses objectifs antiterroristes et sécuritaires. On l'accuse d'avoir jeté la suspicion sur les communautés musulmanes en menant de front travail communautaire et service de renseignement. D'autant plus que Prevent se destine à lutter contre l'extrémisme islamique sans se préoccuper d'autres formes d'extrémisme comme celui de l'extrême droite. Enfin, il est critiqué pour avoir omis toute référence à la nécessité de lutter contre la pauvreté et la marginalisation.

La donne risque de changer radicalement, cependant, depuis l'élection du gouvernement de coalition en 2010. Le discours du Premier ministre David Cameron au sommet européen de Munich le 5 février 2011 a clairement pris pour cible les musulmans, promettant un changement radical dans leur participation au dialogue avec les instances gouvernementales. Il critique les groupes qui "bénéficient de généreux financements publics alors qu'ils font peu pour combattre l'extrémisme" et il annonce que le gouvernement cessera "de financer ou de s'afficher dans des débats avec des organisations qui tout en étant non violentes font sans aucun doute parfois partie du problème" (The Guardian, 7 février 2011). De surcrồt, le gouvernement britannique élu en 2010 a mis fin au programme Prevent, si bien que les associations musulmanes ont perdu cette source de financement. Tout aussi grave, le programme d'austérité général qui supprime la majeure partie des financements aux associations alors que celles-ci sont le socle de la mobilisation et de la capacité d'action des musulmans.

\section{Conclusion}

Depuis la révolution iranienne de 1979, l'islam s'est imposé sur la scène internationale du nouveau millénaire. Les musulmans jouissent en Grande-Bretagne d'une reconnaissance inégalée ailleurs en Europe, mais ils figurent aujourd'hui parmi les minorités ethniques les plus discriminées et les plus désavantagées. En outre, ils sont en butte aux préjugés qui confondent islam et terrorisme et aux répercussions des politiques antiterroristes. Tout d'abord, il est indéniable que s'est instaurée en GrandeBretagne une institutionnalisation très avancée de l'islam, tant par les politiques qui l'ont pris en compte que par la participation active des populations musulmanes ${ }^{(17)}$. 
L'islam a pignon sur rue dans l'espace public. Il semble que le gouvernement travailliste, tout en décriant les travers du multiculturalisme, ait continué de l'appliquer sous sa forme pluriconfessionnelle en dialoguant avec les leaders musulmans au niveau local par l'intermédiaire des municipalités, comme au niveau national.

Par ailleurs, la guerre contre la "terreur" ainsi que les mesures antiterroristes en Grande-Bretagne vont à l'encontre de la participation des musulmans, car elles ne peuvent qu'accentuer l'islamophobie, la discrimination et la marginalisation des populations musulmanes, déjà fortement désavantagées. Cette politique risque de conduire à un repli des musulmans vers leurs communautés pour y rechercher une protection. Paradoxalement, c'est cette politique même qui a offert le meilleur vecteur de participation à certains jeunes musulmans, et tout particulièrement aux femmes musulmanes, par leur adhésion à l'opposition de gauche et au mouvement contre la guerre. Au demeurant, la politique du gouvernement de coalition de David Cameron risque de mettre en péril la place que se sont faite les populations musulmanes dans la société britannique.

\section{Notes}

1. Je remercie Florence Hulak qui a revu le format de cet article.

2. Les chiffres de cette section ont été obtenus dans Ceri Peach, "Muslims in the UK", in Tahir Abbas (dir.), Muslim Britain: Communities Under Pressure, London, Zed Books, 2005, pp. 18-19;21-20; 25 et 28.

3. Les chiffres ci-dessus ont été obtenus dans Ceri Peach, "Muslims in the UK", op. cit.; David Owen, "Demographic Profiles and Social Cohesion of Minority Ethnic Communities in England and Wales", in Community, Work and Family, vol. 9, n³ 3, London, Taylor and Francis, 2006, pp. 267 et 269 ; David Owen, "Profile of Black and Minority Ethnic Groups in the UK", background paper for a seminar on "Understanding and Tackling Ethnic Minorities in Health", University of Warwick, $24^{\text {th }}$ January 2006 (ESRC seminar series: "Migration, Transnational Links and Life-Course Influences on Health"); Tahir Abbas (dir.), Muslim Britain: Communities Under Pressure, London, New York, Zed Books, 2005.

4. Bhikhu Parekh, The Social Logic of Pluralism, London, CRE, 1990.

5. John Rex and Sally Tomlinson, Colonial Immigrants in a British City: A Class Analysis, London, Routledge \& Kegan Paul, 1979.

6. Danièle Joly, Britannia's Crescent: Making a place for Muslims in British Society, Aldershot, Avebury, 1995.

7. Danièle Joly, Blacks and Britannity, Aldershot, Ashgate, 2001.

8. James Arthur Beckford \& Sophie Gilliat, Religion in Prison: Equal Rites in a Multi-Faith Society, Cambridge, Cambridge University Press, 1998.

9. Jorgen Nielsen, Towards a European Islam. Basingstoke, Macmillan, 1999.

10. Muslim Council of Britain, "About MCB", 8 juin 2012, http://www.mcb.org.uk/aboutmcb.php.

11. Deen Siddiqui et al.,"Why Muslims Should Vote for a Labour Government", in The Guardian, 19 avril 2005.

12. Jessica Jacobson, "Religion and Ethnicity: Dual and Alternative Sources of Identity Among Young Pakistanis", in Ethnic and Racial Studies, vol. 2, n 20, 1997, pp. 238-256.

13. Danièle Joly, L'Émeute, Paris, Denoël, 2007.

14. European Monitoring Centre on Racism and Xenophobia, "The Impact of 7 July 2005 London Bomb Attacks on Muslim Communities in the UK", Vienna, November 2005, p. 19.

15. Home Office, "Preventing Extremism Together", working group reports, August-September 2005.

www.communities.gov.uk/pub/16/PreventingExtremismTogetherworkinggroupreportAugOct2005_id1502016.Pdf.

16. The Guardian, 6 août 2005.

17. James Arthur Beckford, Danièle Joly et Farhad Khosrokhavar, Challenge and Change: Muslims in French and British prisons, Basingstoke, Macmillan-Palgrave, 2005. 